\title{
Macroscopic Model of an Abrasive Tool
}

\author{
Nikolay Nosov ${ }^{1}$, Roman Grishin ${ }^{1, *}$, Roman Ladyagin $^{1}$, Vladimir Rodionov ${ }^{1}$ and \\ Yaroslav Gordienko ${ }^{1}$ \\ ${ }^{1}$ Samara state technical university, Russia
}

\begin{abstract}
When designing an abrasive tool, it is necessary to know the most important properties of the abrasive grain-bond system. The greatest interest is the study of the physical and mechanical characteristics of the abrasive tool: hardness, density, bending strength, modulus of elasticity, Poisson's ratio, coefficient of thermal expansion, thermal properties, etc. There are extensive experimental data on mechanical testing of abrasive tools in which a number of empirical formulas have been obtained to determine the physical and mechanical characteristics of an abrasive tool (grain size, hardness, structure, grain and bond grade, etc.). The article is based on core several assumptions and hypotheses that determine the limits of applicability of the results obtained, within which the theory has a complete and complete model of the behavior of the abrasive tool.
\end{abstract}

\section{Introduction}

Theoretical prediction of the macroscopic properties of an abrasive tool is one of the urgent problems of tool production, the successful solution of which will allow obtaining acceptable estimates of the limiting state of an abrasive tool, as well as describing the processes of static and dynamic loading during grinding [1,2]. The mechanical properties of an abrasive tool are not used in our daily practice. For a theoretical description of the properties of a ceramic-bonded abrasive tool, it is necessary to classify these materials as composite materials [3-5] and describe their structure [6]. Taking into account the peculiarities of the heterogeneous structure of the abrasive tool, it can be divided into 3 geometric types:

1. These are materials with a single-phase system, including only abrasive grains located anisotropically, with different orientations of planes and axes of symmetry.

2. Abrasive tools characterized by two phases abrasive grains and pores that are continuous in structure. The geometric interpretation of such materials is that they are mutually penetrating meshes of pores and grains.

3 . These types of abrasive materials are variants of one common case, which include grains, bond, and pores. The structure of such materials is a continuous matrix with inclusions of grains and pores of various shapes.

When describing such a structure of an abrasive tool, two cases are possible:

\footnotetext{
* Corresponding author: konferencia-isap@yandex.ru
} 
- structures with a small fraction of inclusions, in which the grains are removed from each other, and the pores are practically absent, these include abrasive pastes and suspensions;

- structures with different grain fractions and ligaments and pore formation, which is typical for a wide class of abrasive tools on ceramic, bakelite and vulcanite ligaments.

Designing an abrasive tool with the required physical and mechanical properties is a complex task that can be accomplished in two ways [7-10].

The first way consists in the sequential selection of the structure and materials of the components of the abrasive tool, their manufacture, followed by the determination of the physical and mechanical properties. This path leads to large material costs associated with the creation of a production base, the development of new technologies for the manufacture of abrasive tools without reference to existing technologies.

The second way is to study the influence of the mechanical characteristics of the components and the geometric structure of the abrasive tool on its macroscopic properties, which, in our opinion, is more acceptable. Within the framework of the mechanics of composite materials, an abrasive tool can be represented as a continuous inhomogeneous medium formed by two or more components with different deformation and strength characteristics.

\section{Statement of the research problem.}

Let the elastic medium occupy the volume $\mathrm{V}$ bounded by the surface $\mathrm{S}$. The value, $V=\sum_{n=1}^{3} V_{k}$ where $\mathrm{V}_{1}$ is the volume of abrasive grains, $\mathrm{V}_{2}$ is the volume of the matrix (bond) and $\mathrm{V}_{3}$ is the volume of pores, then their volume concentrations are equal $C_{1}=\frac{V_{1}}{V}$; $C_{2}=\frac{V_{2}}{V}$ and $C_{3}=\frac{V_{3}}{V}$. All components in the abrasive tool are considered elastic and are connected to each other with perfect adhesion, each of which corresponds to its own physical and mechanical properties.

The defining relations for the macro-deformations $S_{i j}$ and the macro-stresses of the abrasive tool components are given by the Hooke equations:

$$
\begin{aligned}
& S_{i j}=2 \mu_{s} \cdot e_{i j} ; \\
& \sigma_{i j}=3 K_{s} \cdot E_{p p},
\end{aligned}
$$

where $\mu_{\mathrm{s}}$ and $\mathrm{K}_{\mathrm{s}}$ are respectively the shear and volume modules of the s-th component (Lamé parameter); $\mathrm{E}_{\mathrm{pp}}$ - components of strain tensors; $\mathrm{e}_{\mathrm{ij}}$ - volumetric concentrations of components.

Here $S_{i j}=\sigma_{i j}-\frac{1}{3} \cdot \delta_{i j} \cdot \sigma_{p p} ; e_{i j}=E_{i j}-\frac{1}{3} \cdot \delta_{i j} \cdot \varepsilon_{p p}$,

where $E_{p p}=\sum_{i=1}^{3} E_{i j}, a \delta_{i j}=\left\{\begin{array}{l}1-\bar{r} \in V_{s} \\ 0-\bar{r} \in V_{s}\end{array}\right.$ at $S=\overline{1,3}$

Since real grains and pores have different shapes, the geometric structure of an abrasive tool is described by the theory of random fields. The structure of such a heterogeneous environment can be described by a set of indicator functions 


$$
\aleph_{S}(\vec{r})=\left\{\begin{array}{l}
1-\bar{r} \in V_{s} \\
0-\bar{r} \in V_{s}
\end{array} \text { at } S=\overline{1,3}\right.
$$

each of which is equal to 1 in the volume $\mathrm{V}$ and 0 outside this volume, that is. The system of indicator functions allows us to write equation (2) in the form

$$
\begin{aligned}
& S_{i j}(\vec{r})=2 \cdot \sum_{i=1}^{3} \mu_{s} \cdot \aleph_{s}(\vec{r}) \cdot e_{i j}(\vec{r}), \\
& \sigma_{p p}(\vec{r})=3 \cdot \sum_{i=1}^{3} K_{s} \cdot \aleph_{s}(\vec{r}) \cdot E_{p p}(\vec{r}) .
\end{aligned}
$$

Moreover, the volume ratios obey the condition $\aleph_{1}+\aleph_{2}+\aleph_{3}=1$ or $\aleph_{1}=1-\aleph_{2}-\aleph_{3}$

Taking into account the conditions for the existence of indicator functions, we write equation 3 in the form

$$
\begin{aligned}
& S_{i j}(\vec{r})=2 \mu_{1} \cdot e_{i j}(\vec{r})+2 \cdot \sum_{S=2}^{3}\left[\mu_{s}\right] \cdot \aleph_{s}(\vec{r}) \cdot e_{i j}(\vec{r}), \\
& \sigma_{p p}(\vec{r})=3 K_{1} \cdot E_{p p}(\vec{r})+3 \cdot \sum_{S=2}^{3}\left[K_{s}\right] \cdot \aleph_{s}(\vec{r}) \cdot E_{p p}(\vec{r}) .
\end{aligned}
$$

To solve this system of equations (4), we make several assumptions.

\section{Assumptions}

\section{Assumption 1}

Consider a local law, which is the averaging mechanism and replace $\left\langle e_{i j}\right\rangle \rightarrow\left\langle e_{i j}\right\rangle_{s}$ for $\left\langle\varepsilon_{i j}\right\rangle$ and $\left\langle\varepsilon_{p p}\right\rangle \rightarrow\left\langle\varepsilon_{p p}\right\rangle_{s}$ for $\left\langle\sigma_{p p}\right\rangle_{s}$, then we get $\left\langle S_{i j}\right\rangle=2 \mu_{1} \cdot\left\langle e_{i j}\right\rangle+2 \cdot \sum_{S=2}^{3}\left[\mu_{s}\right] \cdot \aleph_{s}(\vec{r}) \cdot\left\langle e_{i j}\right\rangle_{s}$, $\left\langle\sigma_{p p}\right\rangle=3 K_{1} \cdot\left\langle E_{p p}\right\rangle+3 \cdot \sum_{S=2}^{3}\left[K_{s}\right] \cdot \aleph_{s}(\vec{r}) \cdot\left\langle E_{p p}\right\rangle_{s}$ $\sigma_{i j}(\vec{r})=2 \mu(\vec{r}) \cdot \varepsilon_{i j}(\vec{r})+\delta_{i j} \cdot K(\vec{r}) \cdot \varepsilon_{p p}(\vec{r}) ;$ where $\mu(\vec{r})=\sum_{S=2}^{3} \mu_{s} \cdot \aleph_{S}(\vec{r})=\mu_{1} \cdot \aleph_{1}+\mu_{2} \cdot \aleph_{2} ; K(\vec{r})=\sum_{S=2}^{3} K_{s} \cdot \aleph_{S}(\vec{r})=K_{1} \cdot \aleph_{1}+K_{2} \cdot \aleph_{2}$.

\section{Assumption 2}

All physical and geometric quantities are assumed to be statically homogeneous with ergodic fields and are replaced by averaging over the corresponding characteristic volume

$\left.f(\vec{r})\right|_{r \in S}=\left.\langle f\rangle \Leftrightarrow f_{(\vec{r})}^{\prime}\right|_{S}=0$

where $\langle f\rangle=\frac{1}{V} \cdot \int_{V} f(r) d r$ and $\left\langle f_{S}\right\rangle=\frac{1}{V_{S}} \cdot \int_{V_{S}} f(r) d r$

for $\langle f\rangle=$ const, there is an ergodicity condition.

Averaging equation (5) over the total volume of the abrasive tool gives the following system of equations: 


$$
\begin{aligned}
& \left\langle S_{i j}\right\rangle=2 \mu_{1} \cdot\left\langle e_{i j}\right\rangle+2 \cdot \sum_{S=2}^{3} C_{s} \cdot\left[\mu_{s}\right] \cdot\left\langle e_{i j}\right\rangle_{s} ; \\
& \left\langle\sigma_{p p}\right\rangle=3 K_{1} \cdot\left\langle E_{p p}\right\rangle+3 \cdot \sum_{S=2}^{3} C_{s} \cdot\left[K_{s}\right] \cdot\left\langle\varepsilon_{p p}\right\rangle_{s} ;
\end{aligned}
$$

where $C_{s}=V_{s} \cdot V^{-1}$ is the volumetric content of the components in the abrasive tool.

\section{Assumption 3}

To determine the effective Hooke's law, it is necessary to express the deformation $\left\langle\varepsilon_{i j}\right\rangle_{s}$, in terms of the macrodeformation $\left\langle\varepsilon_{i j}\right\rangle$. To do this, we use the well-known ratio [6] $\left\langle E_{i j}\right\rangle_{S}=\left\langle E_{i j}\right\rangle+C_{S}^{-1} \cdot\left\langle\aleph_{S}^{\prime} \cdot E_{i j}^{\prime}\right\rangle$

Here, the strokes indicate the fluctuations of the quantities in volume $\mathrm{V}$.

The deformations set of equations of the abrasive tool with respect to fluctuations will have the form:

$$
\begin{aligned}
& \left\langle S_{i j}^{\prime}\right\rangle=2 \mu_{1} \cdot\left\langle e_{i j}^{\prime}\right\rangle(\vec{r})+2 \cdot \sum_{S=2}^{3}\left[\mu_{s}\right] \cdot \aleph_{s} \cdot \aleph_{s}^{\prime} \cdot\left\langle e_{i j}\right\rangle_{s} ; \\
& \left\langle\sigma_{p p}^{\prime}\right\rangle=3 K_{1} \cdot\left\langle E_{p p}^{\prime}\right\rangle(\vec{r})+3 \cdot \sum_{S=2}^{3}\left[K_{s}\right] \cdot \aleph_{s} \cdot \aleph_{s}^{\prime} \cdot\left\langle E_{p p}\right\rangle_{s} .
\end{aligned}
$$

Let's write the equation of equilibrium:

$$
\begin{gathered}
\sigma_{i j, j}^{\prime}(\vec{r})=0 \\
\text { where } \sigma_{12,3}=\frac{\partial \sigma_{12}}{\partial x_{3}} \text {; for } i=1 \frac{\partial \sigma_{11}}{\partial x_{1}}+\frac{\partial \sigma_{12}}{\partial x_{2}}+\frac{\partial \sigma_{13}}{\partial x_{3}}=0 \\
i=2 \frac{\partial \sigma_{21}}{\partial x_{1}}+\frac{\partial \sigma_{22}}{\partial x_{2}}+\frac{\partial \sigma_{23}}{\partial x_{3}}=0 .
\end{gathered}
$$

The value of $\aleph_{s}$ in equations (6) characterizes the degree of connectivity of the constituent components $\min \leq \aleph_{S} \leq \max$. In this case, the value $\aleph_{S} \rightarrow$ max corresponds to the spherical shape of the inclusions (maximum connectivity), and the minimum - to the plate.

\section{Macroscopic model}

Using the isotropy conditions for indicator functions $\aleph_{s}^{\prime}(r)$, we obtain Hooke's macroscopic law:

$$
\begin{aligned}
& \left\langle S_{i j}\right\rangle=2 \mu * \cdot\left\langle\varepsilon_{i j}\right\rangle, \\
& \left\langle\sigma_{p p}\right\rangle=3 K^{*} \cdot\left\langle\varepsilon_{p p}\right\rangle .
\end{aligned}
$$

Here $\mathrm{K}^{*} \mathrm{u} \mu^{*}$ effective modules of the composite 


$$
\begin{aligned}
& \mu^{*}=\xi^{-1} \cdot \sum_{j=1}^{3} \mu_{s} \cdot C_{s} \cdot a_{s}, \\
& K^{*}=\eta^{-1} \cdot \sum_{S=1}^{3} K_{s} \cdot C_{s} \cdot b_{s} ;
\end{aligned}
$$

$$
\begin{aligned}
& \text { where } \xi=\sum_{S=1}^{3} C_{s} \cdot a_{s} ; \eta=\sum_{S=1}^{3} C_{s} \cdot b_{s} \\
& a_{s}=\left[1+\alpha_{1} \cdot\left(m_{s}-1\right) \cdot \aleph_{s}\right]^{-1} ; b_{s}=\left[1+\gamma_{1} \cdot\left(q_{s}-1\right) \cdot \aleph_{s}\right]^{-1} \\
& m_{s}=\frac{\mu_{s}}{\mu_{1}} ; \quad q_{s}=\frac{K_{s}}{K_{1}} ; \alpha_{1}=\frac{2}{15} \cdot \frac{4-5 v_{1}}{1-v_{1}} ; \alpha_{1}=\frac{1}{3} \cdot \frac{1+v_{1}}{1-v_{1}} ;
\end{aligned}
$$

$v_{1}$ - the Poisson's ratio of the matrix.

These relations were a general model of a chaotically reinforced three-component abrasive tool and allow us to solve the problem for a three-phase porous medium, in which the first component is the abrasive grains $\mathrm{s}=1$, the second $\mathrm{s}=2$ is bond-matrix, and the third $\mathrm{s}=3$ - pores. Given that $\mu_{\mathrm{s}}=\mathrm{K}_{\mathrm{s}}=0$, and $C_{r}=\frac{C_{2}}{C_{1}}$ or $C_{1}=\frac{1-C_{3}}{1+C_{r}}$, we find

$$
\mu_{s}=\frac{E_{s}}{2 \cdot\left(1+v_{s}\right)} ; K_{s}=\frac{E_{s}}{3 \cdot\left(1+2 v_{s}\right)} \text {. }
$$

Thus, the effective shear modulus $\mu^{*}$ and the effective volume modulus $\mathrm{K}^{*}$ are determined by the formula

$$
\begin{aligned}
& \mu^{*}=\frac{1}{\xi} \cdot\left(\mu_{s} \cdot C_{2}+\mu_{s} \cdot C_{1} \cdot a_{s}\right) ; \\
& K^{*}=\frac{1}{\eta} \cdot\left(K_{s} \cdot C_{2}+K_{s} \cdot C_{1} \cdot b_{s}\right) ;
\end{aligned}
$$

where $a_{\mathrm{s}}$ and $b_{\mathrm{s}}$ are structural coefficients describing the shape of grains and pores, as well as the nature of their connectivity

$$
\begin{gathered}
a_{1}=1 ; a_{2}=\frac{1}{1+\alpha_{1} \cdot\left(m_{2}-1\right) \cdot \aleph_{2}} ; a_{3}=\frac{1}{1+\alpha_{1} \cdot \aleph_{3}} ; \\
b_{1}=1 ; b_{2}=\frac{1}{1+\gamma_{1} \cdot\left(q_{2}-1\right) \cdot \aleph_{2}} ; a_{3}=\frac{1}{1+\gamma_{1} \cdot \aleph_{3}} .
\end{gathered}
$$

The values $\xi$ and $\eta$ characterize the volume ratios of the components included in the abrasive tool $\xi=C_{2}+C_{1} \cdot a_{2}+C_{3} \cdot a_{3}, \eta=C_{2}+C_{1} \cdot b_{2}+C_{3} \cdot b_{3}$.

The values $\aleph_{2}$ and $\aleph_{3}$ depend on the connectivity of the grains and pores. The grain connectivity is not a geometric, but a technological property of the structure of an abrasive tool, and topological networks are used for its quantitative description, in which closed surfaces (grains) are represented by nodes, and the contacts between them are branches connecting the corresponding nodes. For abrasive tools on ceramic bonds, the branches are the bridges of the bond between the grains that are formed at the time of sintering.

Thus, it is possible to calculate the effective modulus of elasticity (Young's) $\mathrm{E}^{*}$ and the Poisson's ratio $v^{*}$ of an abrasive tool on ceramic bonds:

$$
\begin{aligned}
& E^{*}=\frac{9 \mu^{*} \cdot K^{*}}{3 K^{*}+\mu^{*}}, \\
& v^{*}=\frac{3 K^{*}-2 \mu^{*}}{6 K^{*}+2 \mu^{*}} .
\end{aligned}
$$


On the basis of the proposed mathematical model, it is possible, to a certain extent, to determine the physical and mechanical properties of an abrasive tool on ceramic bonds. The effective modulus of elasticity and Poisson's ratio of the abrasive tool change depending on the elastic properties of the abrasive grain and bond, volumetric ratios and connectivity, of the incoming components. To simplify the calculations of the elastic characteristics of the abrasive tool, a computer program has been developed. Theoretical studies of elastic contacts $\mathrm{E}^{*}$ and $v^{*}$ were carried out in the entire range of characteristics: grain size, structure, hardness, grain grade and binding. For the convenience of analyzing the results of the study, we will take as an argument not the absolute values, but their ratios, that is, $\frac{E^{*}}{E_{1}}$ and $\frac{v^{*}}{v_{1}}$, where $\mathrm{E}_{1}$ is the elastic modulus of the grain material, and $v_{1}$ is the Poisson's ratio of the grain material.

Fig. 1 show the results of calculating the elastic characteristics of an abrasive tool for the entire range of porosity and structure with a grain elastic modulus varying from $\mathrm{E}_{1}=$ $300 \mathrm{GPa}$ to $\mathrm{E}_{1}=400 \mathrm{GPa}$ and Poisson's ratio $v_{1}=0.25-0.3$

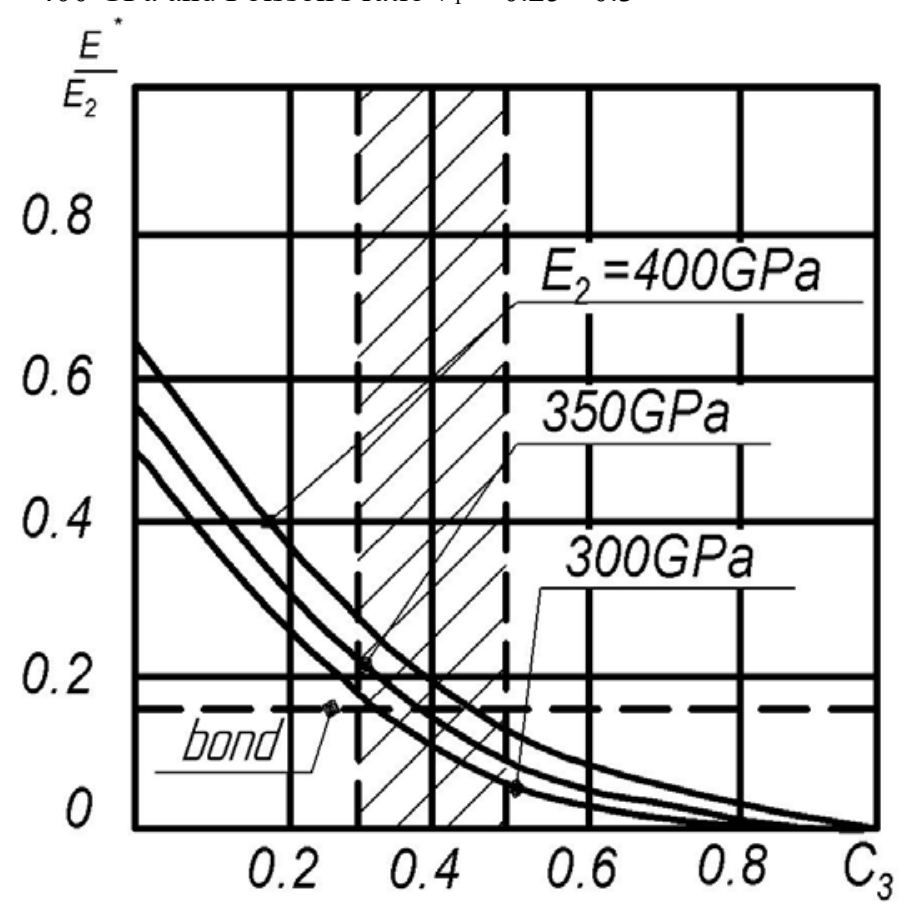

Fig.1. Influence of the total porosity and porosity of the abrasive material (shaded area) on the relative effective modulus of elasticity at $\mathrm{E}_{1}=350 \mathrm{GPa}$

Studies have shown that the obtained dependences are not linear. In addition, when the porosity is equal to one, all of them converge to zero.

\section{Conclusion}

It was found that the shape of the grains and pores affects the physical and mechanical properties of the abrasive tool. The maximum values of the macroscopic properties are obtained when the pores and grains are spherical. In real tools, an increase in the coefficient 
of sphericity of grains leads to a decrease in the coefficient of sphericity of pores, so it is important to study their mutual influence.

\section{References}

1. N.V. Nosov, A.V. Rozhnyatovskiy, Investigation of the physical and mechanical properties of SHS-corundum, Physical processes when cutting metals,pp. 117-121 (1996)

2. N.V. Nosov, A.V. Rozhnyatovskiy, Physical and mechanical properties of abrasive tools, Finishing and hardening technology in mechanical engineering, pp. 48-49 (1994).

3. Christensen R. Introduction to the theory of composites (M: mir, 1982).

4. Shermergor T.D. Theory of elasticity of micro-inhomogeneous media. (M: Nauka, 1977).

5. A.M. Caprinosa, Composite materials (Kiev: Naukova Dumka, 1985).

6. N.V. Nosov, A new approach to the process of forming the structure of abrasive tool, Processes of abrasive treatment, abrasive tools and materials, pp. 19-21 (Polygraphist 1997).

7. N.V. Nosov, L.A. Saraev, V.A Sakhabiev, Mathematical model of an abrasive tool made of SHS materials, Bulletin of Samara State Technical University,v.1, pp. 124130 (1994).

8. N.V. Nosov, Phenomenological model of the abrasive tool working surface, Bulletin of Samara State Technical University. v.10, pp. 141-146 (2000).

9. N.V. Nosov, Yu.N. Kuzmin, A.V. Rozhnyatovskiy, Modeling of an abrasive tool Modern problems of automation of mechanical engineering, pp. 14-15 (1995)

10. N.V. Nosov, M.Yu. Rakityansky, Model of an abrasive tool without a bond, Thermophysical and technological aspects of quality management in mechanical engineering, pp. 303-304 (2005). 